STAATSMINISTERIUM

DER FINANZEN

Freistaat

SACHSEN

\title{
Sonderausgaben und
}

\section{Außergewöhnliche Belastungen}

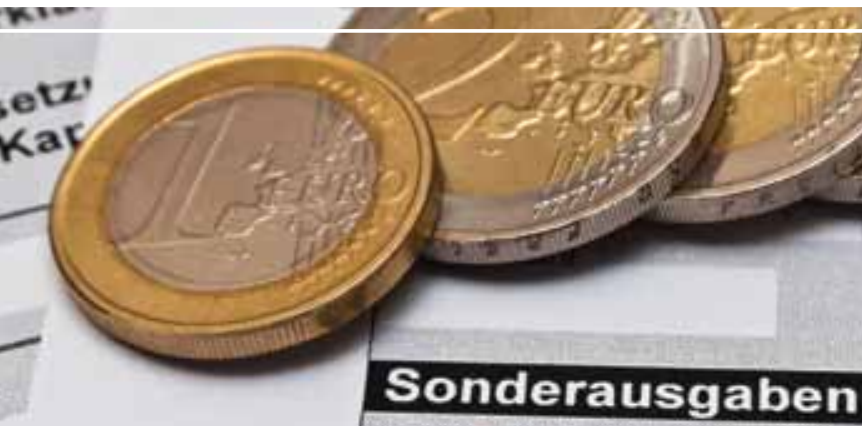

Gezablte Versorgungsleistungen

Wohnort

Rechtsgrund, Datum des Vertrags

Rechtsgrund, Datum des Vertrags

Dauernde Lasten

39 Ausgleichszahlungen im Rz. Rec schuldrechtlichen Versorgury 


\section{Vorwort}

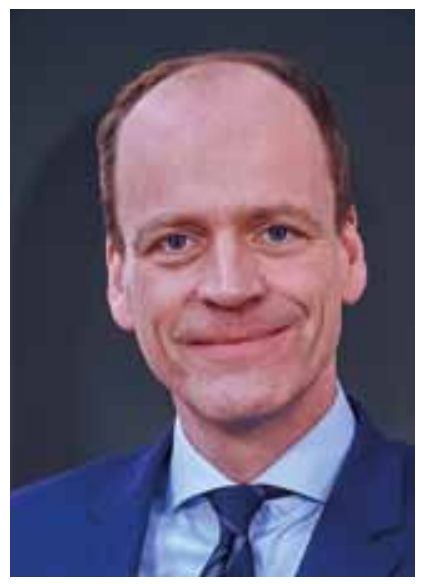

Sehr geehrte Leser,

gleiches Ritual - neues Jahr: mit der Abgabe Ihrer Einkommensteuererklärung können Sie die Möglichkeit zur Steuererstattung nutzen. Neben den Ausgaben im Zusammenhang mit der beruflichen Tätigkeit, den sogenannten Werbungskosten, kann aber auch für andere Aufwendungen eine Steuerminderung in Betracht kommen. Dies ist beispielsweise für Beiträge zu Versicherungen oder Krankheitskosten der Fall.

In dieser Broschüre erfahren Sie, welche privaten Ausgaben als Sonderausgaben oder als außergewöhnliche Belastungen abziehbar sind. Dabei wird auch auf die neue Rechtsprechung zur stufenweisen Ermittlung der zumutbaren Belastung sowie auf die Abzugsmöglichkeit für Spenden eingegangen.

Mit dieser Broschüre erhalten Sie einen guten Überblick über die einschlägigen Vorschriften. Sollten darüber hinaus Fragen offen bleiben, geben die Mitarbeiterinnen und Mitarbeiter unserer Finanzämter gern Auskunft. Dafür ist das Info-Telefon der Finanzämter unter der Telefonnummer $035179997888^{1}$ von Montag bis Donnerstag von 8 bis 17 Uhr und am Freitag von 8 bis 12 Uhr erreichbar.

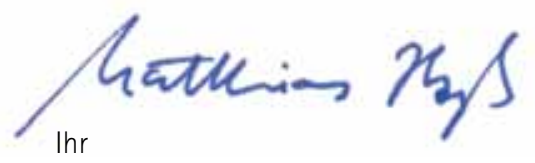

Dr. Matthias $\mathrm{Haß}$

Sächsischer Staatsminister der Finanzen

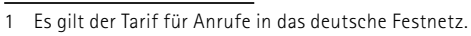




\section{Inhaltsverzeichnis}

1. Sonderausgaben .......................................................................................................................... 3

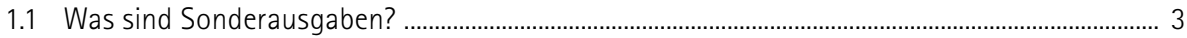

1.2 Vorsorgeaufwendungen ............................................................................................................ 3

1.2.1 Altersvorsorgeaufwendungen ......................................................................................... 4

1.2.2 Sonstige Vorsorgeaufwendungen (Versicherungsbeiträge) ................................................ 5



1.3 Riesterförderung ........................................................................................................................... 8

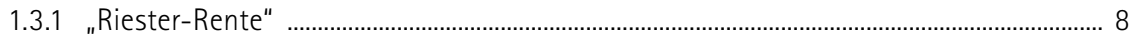

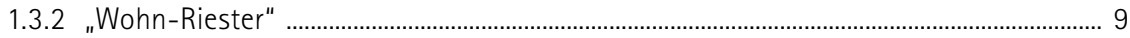

1.4 Unterhaltsleistungen an den geschiedenen oder dauernd getrennt lebenden Ehegatten ............ 10

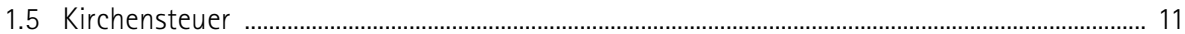

1.6 Kinderbetreuungskosten ............................................................................................................. 11

1.7 Aufwendungen für die eigene Berufsausbildung ......................................................................... 12



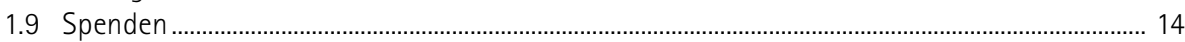

1.10 Sonderausgaben-Pauschbetrag/Vorsorgepauschale................................................................... 15

2. Außergewöhnliche Belastungen ................................................................................. 17

2.1 Was sind außergewöhnliche Belastungen? ............................................................................... 17

2.2 Außergewöhnliche Belastungen allgemeiner Art ....................................................................... 17

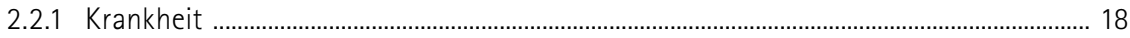

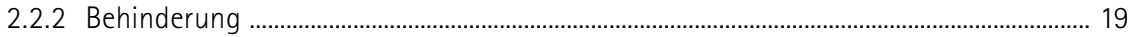

Tatsächliche Aufwendungen .............................................................................................. 19

Pauschbetrag für behinderte Menschen ............................................................................ 20

2.2.3 Pflegebedürftigkeit ............................................................................................................ 21

Abziehbare Aufwendungen ....................................................................................................... 21

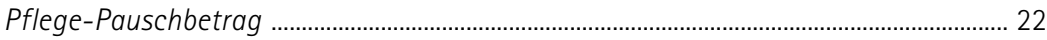

2.2.4 Hinterbliebenen-Pauschbetrag ............................................................................................ 23

2.2.5 Weitere außergewöhnliche Belastungen ............................................................................. 23

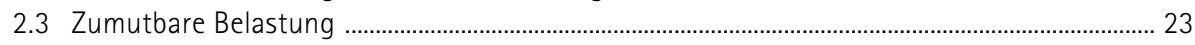

2.4 Außergewöhnliche Belastungen in besonderen Fällen .............................................................. 24

2.4.1 Unterhaltsaufwendungen ................................................................................................. 24

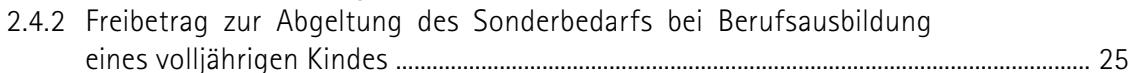

3. Berücksichtigung beim Lohnsteuerabzug .................................................................. 26



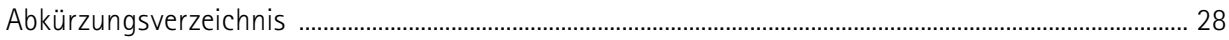




\section{Sonderausgaben}

\subsection{Was sind Sonderausgaben?}

Sonderausgaben sind private Ausgaben für bestimmte Kosten der Lebensführung, die vom Staat ausnahmsweise steuerlich begünstigt werden. Das Einkommensteuergesetz enthält eine abschließende Aufzählung der Sonderausgaben (§§ $10 \mathrm{ff}$. des Einkommensteuergesetzes).

Für Sonderausgaben gilt der Grundsatz, dass es sich bei den Aufwendungen nicht um Betriebsausgaben oder Werbungskosten handeln darf.

Sonderausgaben sind in der Einkommensteuererklärung auf Seite 2 des Mantelbogens sowie in den Anlagen „Vorsorgeaufwand", "Kind" und "AV" ("Riester-Rente") zu erklären. Die bundeseinheitlichen Vordrucke samt Anleitung zur Einkommensteuererklärung sind beim Finanzamt erhältlich bzw. stehen im Internet unter www.steuern.sachsen.de $\rightarrow$ Vordrucke $\rightarrow$ Bürger $\rightarrow$ Formular-Management-System der Bundesfinanzverwaltung oder unter www.formulare-bfinv.de zum Download zur Verfügung.

Abzugsberechtigt ist derjenige, der zur Zahlung verpflichtet ist und die entsprechenden Aufwendungen auch selbst entrichtet.

Abzugszeitpunkt ist das Kalenderjahr, in dem die Sonderausgaben geleistet werden.
Die Abzugshöhe bestimmt sich grundsätzlich nach den tatsächlichen Zahlungen innerhalb eines Kalenderjahres abzüglich der im gleichen Kalenderjahr erstatteten oder gutgeschriebenen Beträge der gleichen Art (z. B. erstattete Kirchensteuer, rückvergütete Versicherungsbeiträge).

Im Folgenden werden einzelne Sonderausgaben, die häufig vorkommen, vorgestellt:

\subsection{Vorsorgeaufwendungen}

Als Vorsorgeaufwendungen können Ausgaben für die Altersvorsorge (z.B. Beiträge zur gesetzlichen Rentenversicherung $\rightarrow$ Nr. 1.2.1) und sonstigen Vorsorgeaufwendungen (z.B. Beiträge zur Kranken- und Pflegeversicherung sowie weitere Versicherungsbeiträge $\rightarrow$ Nr. 1.2.2) grundsätzlich bis zu bestimmten Höchstbeträgen steuermindernd abgezogen werden. Daneben ist eine steuerliche Förderung der Beiträge für eine zusätzliche Altersvorsorge (sog. Riester-Verträge) möglich ( $\rightarrow$ Nr. 1.3).

Der Abzug von Vorsorgeaufwendungen ist insbesondere an folgende Voraussetzungen gebunden:

1. Die Vorsorgeaufwendungen dürfen nicht in unmittelbarem wirtschaftlichen Zusammenhang mit steuerfreien Einnahmen (z. B. steuerfreier Arbeitslohn bei Auslandstätigkeit) stehen. Eine Ausnahme gilt für Vorsorgeaufwendungen, soweit 
a) sie in unmittelbarem wirtschaftlichen Zusammenhang mit in einem Mitgliedstaat der Europäischen Union oder einem Vertragsstaat des Abkommens über den Europäischen Wirtschaftsraum erzielten Einnahmen aus nichtselbständiger Tätigkeit stehen,

b) diese Einnahmen nach einem Abkommen zur Vermeidung der Doppelbesteuerung im Inland steuerfrei sind und

c) der Beschäftigungsstaat die Vorsorgeaufwendungen steuerlich nicht berücksichtigt.

\section{Die Vorsorgeaufwendungen müssen an}

- Versicherungsunternehmen, die ihren Sitz oder ihre Geschäftsleitung in der Europäischen Union oder einem anderen Vertragsstaat des Europäischen Wirtschaftsraumes haben und das Versicherungsgeschäft im Inland betreiben dürfen oder

Versicherungsunternehmen, denen die Erlaubnis zum Geschäftsbetrieb im Inland erteilt ist oder

- eine berufsständische Versorgungseinrichtung oder

- einen Sozialversicherungsträger oder

- einen Anbieter von Altersvorsorgeverträgen (Riester-Verträgen) oder

- Pensionsfonds, Pensionskassen oder Direktversicherungen

gezahlt werden.

\subsubsection{Altersvorsorgeaufwendungen}

Zu den Altersvorsorgeaufwendungen zählen Beiträge:

zu den gesetzlichen Rentenversicherungen,

zur landwirtschaftlichen Alterskasse,

- zu berufsständischen Versorgungseinrichtungen, die den gesetzlichen Rentenversicherungen vergleichbare Leistungen erbringen, zu zertifizierten Rentenverträgen (sog. „Rürup-Rente" oder Basisrentenverträge),

zu einer betrieblichen Altersversorgung, wenn der Versorgungszusage ein zertifizierter Basisrentenvertrag zugrunde liegt und

zu einer ausländischen gesetzlichen Rentenversicherung.

Neben Beiträgen zu den zertifizierten Basisrentenverträgen-Altersvorsorge (sog. „RürupRente") können auch Beiträge zu zertifizierten Basisrentenverträgen-Erwerbsminderung steuermindernd berücksichtigt werden.

Ein zertifizierter Basisrentenvertrag-Altersvorsorge (sog. „Rürup-Rente") dient dem Aufbau einer eigenen kapitalgedeckten Altersversorgung und muss insbesondere eine lebenslange monatliche Leibrente vorsehen, deren Auszahlung nicht vor Vollendung des 62. Lebensjahres (bei Vertragsabschlüssen vor dem 1. Januar 2012 nicht vor Vollendung des 60. Lebensjahres) erfolgt. Die Ansprüche aus dem Basisrentenvertrag-Altersvorsorge, können ergänzend mit einer Hinterbliebenenabsicherung kombiniert werden (nur Absicherung des Ehegatten/eingetragenen Lebenspartners und der Kinder, für die der Vertragspartner einen Anspruch auf Kindergeld oder einen Kinderfreibetrag hat). Daneben ist in einem gewissen Umfang die Absicherung für den Fall des Eintritts einer Berufsoder Erwerbsminderung ergänzend möglich.

Beiträge zu einem zertifizierten Basisrentenvertrag-Erwerbsminderung sind ab 2014 steuerlich begünstigt. Sie dienen der Absicherung des Risikos der Erwerbsminderung oder der Berufsunfähigkeit. Der Vertrag darf nur die Zahlung einer monatlichen, lebenslangen Rente vorsehen.

Bei den zertifizierten Basisrentenverträgen dürfen die erworbenen Rentenanwartschaften 
weder übertragbar, beleihbar, veräußerbar, vererblich noch kapitalisierbar sein. Auch eine Teilauszahlung des angesparten Vorsorgekapitals ist unzulässig. Die steuerliche Begünstigung von Beiträgen zu einem zertifizierten Basisrentenvertrag ist zudem nur möglich, wenn gegenüber dem Anbieter - in der Regel der Versicherungsgesellschaft - in die Übermittlung der Beitragsdaten an die Finanzverwaltung eingewilligt wurde.

Die Altersvorsorgeaufwendungen sind bis zu einem Höchstbetrag steuerlich zu berücksichtigen. Dieser entspricht dem jeweils gültigen Höchstbeitrag zur knappschaftlichen Rentenversicherung - 2018 beträgt er 23.712 Euro (24,7\% von 96.000 Euro). Bei Zusammenveranlagung verdoppelt sich der Höchstbetrag. Für 2018 sind von den innerhalb des Höchstbetrages geleisteten Altersvorsorgeaufwendungen 86\% als Sonderausgaben abzugsfähig. Dieser Prozentsatz erhöht sich in den nachfolgenden Jahren bis 2025 stufenweise um jährlich $2 \%$.

Bei Arbeitnehmern, die während des ganzen oder eines Teils des Kalenderjahres in der gesetzlichen Rentenversicherung versicherungsfrei sind oder nicht der gesetzlichen Rentenversicherung unterliegen und einen Anspruch auf Altersversorgung erwerben, z.B. Beamte, Richter, Gesellschafter-Geschäftsführer mit Pensionszusage sowie bei Abgeordneten, die ganz oder teilweise ohne eigene Beitragsleistung einen Anspruch auf Altersversorgung erwerben, vermindert sich der vorgenannte Höchstbetrag um einen fiktiven Gesamtbeitrag zur gesetzlichen Rentenversicherung. Dieser wird nach dem jeweils gültigen Beitragssatz zur gesetzlichen Rentenversicherung der Arbeiter und Angestellten und den aus der betreffenden Tätigkeit erzielten steuerpflichtigen Einnahmen ermittelt. 2018 beträgt der maximale Kürzungsbetrag 12.945 Euro (18,6\% von 69.600 Euro).
Beispiel 1:

Herr Schmidt hat im Jahr 2018 einen BruttoarbeitsIohn von 30.000 Euro. Der Arbeitgeber- und Arbeitnehmeranteil zur gesetzlichen Rentenversicherung beträgt jeweils 9,3\% (2.790 Euro). Zusätzlich hat er einen zertifizierten Basisrentenvertrag-Altersvorsorge abgeschlossen und dort Beiträge in Höhe von 1.000 Euro eingezahlt:

Gesamtbeiträge (2.790 Euro +

2.790 Euro + 1.000 Euro)

6.580 Euro

Höchstbetrag

23.712 Euro

anzusetzen

6.580 Euro

davon im Jahr 2018 zu

berücksichtigen $86 \%$

5.659 Euro

abzüglich (steuerfreier

Arbeitgeberanteil)

- 2.790 Euro

Abzugsbetrag

2.869 Euro

Beispiel 2:

wie Beispiel 1, aber Herr Schmidt ist Beamter und erhält Besoldung in Höhe von 30.000 Euro

Gesamtbeiträge

1.000 Euro

Höchstbetrag

23.712 Euro

fiktiver Gesamtrentenbei-

trag (30.000 Euro x 18,6\%=) - 5.580 Euro

gekürzter Höchstbetrag

18.132 Euro

anzusetzen

1.000 Euro

davon im Jahr 2018 zu

berücksichtigen $86 \%$

860 Euro

Abzugsbetrag

860 Euro

\subsubsection{Sonstige Vorsorgeaufwendungen (Versicherungsbeiträge)}

Für die übrigen Sozialversicherungsbeiträge (Kranken-, Pflege- und Arbeitslosenversicherung) und weiteren sonstigen Vorsorgeaufwendungen (z. B. private Haftpflicht- und Risikoversicherungen) gibt es ein separates Abzugsvolumen.

$\mathrm{Zu}$ den sonstigen Vorsorgeaufwendungen zählen insbesondere Beiträge zu: 
gesetzlichen und privaten Kranken- und Pflegeversicherungen,

- Versicherungen gegen Arbeitslosigkeit (gesetzliche Beiträge an die Bundesagentur für Arbeit und Beiträge zu privaten Versicherungen),

- Erwerbs- und Berufsunfähigkeitsversicherungen, die nicht Bestandteil eines Basisrentenvertrages $(\rightarrow$ Nr. 1.2.1) sind,

- Unfallversicherungen, wenn es sich nicht um eine Unfallversicherung mit garantierter Beitragsrückzahlung handelt,

- Haftpflichtversicherungen (Privat-/Tierhalter-/Kfz-, ohne Kaskoanteil) und

- Lebensversicherungen, die nur für den Todesfall eine Leistung vorsehen (Risikolebensversicherungen).

Ferner sind Beiträge zu nachfolgenden Versicherungsverträgen zu berücksichtigen, sofern diese vor dem 1. Januar 2005 begonnen haben und bei denen mindestens ein Versicherungsbeitrag bis zum 31. Dezember 2004 entrichtet wurde:

- Rentenversicherungen ohne Kapitalwahlrecht, bei denen die vorgenannten Voraussetzungen nicht vorliegen,

- Rentenversicherungen mit Kapitalwahlrecht gegen laufende Beitragsleistungen, wenn das Kapitalwahlrecht nicht vor Ablauf von zwölf Jahren seit dem Vertragsabschluss ausgeübt werden kann (max. 88\% der Beiträge),

- Kapitalversicherungen gegen laufende Beitragsleistungen mit Sparanteil, wenn der Vertrag für die Dauer von mindestens zwölf Jahren abgeschlossen wird (max. 88\% der Beiträge).

Nicht als Sonderausgaben abziehbar sind Beiträge zu:

fondsgebundenen Lebensversicherungen,

Hausratversicherungen,

Kaskoversicherungen und

Rechtsschutzversicherungen.
Bei den sonstigen Vorsorgeaufwendungen wird zwischen den steuerlich voll absetzbaren Beiträgen zur sog. Basiskrankenversicherung und zur gesetzlichen Pflegeversicherung einerseits sowie den beschränkt abziehbaren weiteren Vorsorgeaufwendungen andererseits unterschieden. Dies gilt unabhängig davon, ob jemand privat oder gesetzlich versichert ist. Darüber hinaus können insbesondere privat Krankenversicherte zusätzliche Beiträge zur Kranken- und Pflegeversicherung des Ehegatten oder eingetragenen Lebenspartners sowie für mitversicherte Kinder vollständig geltend machen.

Abzugsberechtigt ist derjenige, der die Beiträge als Versicherungsnehmer gezahlt hat. Es ist ohne Bedeutung, wer die versicherte Person ist oder wem die Versicherungsleistungen zufließen. Daneben können Eltern die von ihnen im Rahmen ihrer Unterhaltsverpflichtungen getragenen Beiträge für die Basiskrankenversicherung und gesetzliche Pflegeversicherung ihres Kindes als Sonderausgaben vollständig steuermindernd absetzen, soweit sie für das Kind einen Anspruch auf Kindergeld oder Kinderfreibetrag haben.

Der Abzug der sonstigen Vorsorgeaufwendungen ist grundsätzlich auf 2.800 Euro bzw. 1.900 Euro begrenzt. Er ist davon abhängig, ob die Krankheitskosten oder Beiträge für eine Krankenversicherung ausschließlich aus eigenen Mitteln bestritten oder zumindest Anteile der Krankheitskosten von Dritter Seite übernommen werden. Steuerfreie Leistungen in diesem Zusammenhang sind beispielsweise:

der Arbeitgeberanteil zur Krankenversicherung bei sozialversicherungspflichtigen Arbeitnehmern,

entsprechende Zuschüsse des Arbeitgebers bei freiwillig oder privat krankenversicherten Arbeitnehmern, 
- Beitragsanteile der Träger der gesetzlichen Rentenversicherungen,

- Beihilfeansprüche von Beamten,

- Beiträge der Künstlersozialkasse und

- Beitragserstattungen der Träger von Kindertagespflegeeinrichtungen an Tagespflegepersonen.

Als Höchstbetrag werden:

- 1.900 Euro pro Kalenderjahr bei Personen berücksichtigt, die ganz oder teilweise ohne eigene Aufwendungen einen Anspruch auf vollständige oder teilweise Erstattung oder Übernahme der Krankheitskosten haben oder für deren Krankenversicherung steuerfreie Leistungen erbracht werden,

- 2.800 Euro pro Kalenderjahr bei allen anderen Personen berücksichtigt - z.B. Selbständige, die ihre Krankenversicherung und Krankheitskosten vollständig aus eigenen Mitteln tragen.

Bei zusammen veranlagten Ehegatten/Lebenspartnern ist zunächst für jeden der entsprechende Höchstbetrag zu bestimmen. Gemeinsame Aufwendungen sind dann bis zur Summe der beiden ermittelten Höchstbeträge abziehbar.

Übersteigen die Beiträge zur existenznotwendigen Krankenversicherung (Basiskrankenversicherung) und zur gesetzlichen Pflegeversicherung die vorgenannten Höchstbeträge, sind diese in voller Höhe abziehbar. Weitere sonstige Vorsorgeaufwendungen können dann nicht mehr steuermindernd berücksichtigt werden.

Für Versicherte in der gesetzlichen Krankenversicherung bedeutet dies, dass sie ihre Beiträge grundsätzlich in voller Höhe abziehen können. Erwerben sie mit ihren Beiträgen auch einen Krankengeldanspruch, werden die geleisteten Beiträge pauschal um $4 \%$ gekürzt.
Eigene Beiträge privat Krankenversicherter sind ebenfalls vollständig abziehbar, soweit sie dem Erwerb eines Versicherungsschutzes dienen, der dem der gesetzlichen Krankenversicherung entspricht. Beiträge für eine darüber hinausgehende Versorgung, z. B. Chefarztbehandlung, Einbettzimmer, sowie zur Finanzierung eines Krankengelds gehören hingegen nicht dazu. Die Versicherungsgesellschaft ermittelt den diesbezüglichen Beitragsanteil und teilt diesen mit.

Die Beiträge zur Basiskrankenversicherung und zur gesetzlichen Pflegeversicherung werden nur dann steuermindernd als Sonderausgaben berücksichtigt, wenn gegenüber dem Versicherungsunternehmen oder dem Träger der gesetzlichen Kranken- und Pflegeversicherung in die elektronische Übermittlung der diesbezüglichen Daten eingewilligt wurde. Die Einwilligung zur Datenübermittlung muss jedoch nicht zwangsläufig bei Vertragsbeginn abgegeben werden. Es reicht aus, wenn diese innerhalb einer Frist von zwei Jahren nach Ablauf des betreffenden Beitragsjahres erteilt wird. Die daraufhin vom Versicherungsunternehmen übermittelten Beitragsdaten werden in diesen Fällen auch dann noch steuerlich berücksichtigt, wenn ein bereits bestandskräftiger Einkommensteuerbescheid vorliegt.

\subsubsection{Günstigerprüfung}

Durch die im Jahre 2005 vorgenommene Neuordnung der steuerlichen Berücksichtigung von Vorsorgeaufwendungen können sich in Einzelfällen gegenüber dem bis zum Jahr 2004 geltenden Recht Nachteile ergeben. Zur Vermeidung einer solchen Schlechterstellung wird durch das Finanzamt eine sog. Günstigerprüfung durchgeführt. Im Rahmen dieser Günstigerprüfung werden die Abzugsvolumen verglichen, die sich nach aktuellem Recht und nach dem bis zum Jahr 2004 geltenden Recht für 
den Sonderausgabenabzug von Vorsorgeaufwendungen (Altersvorsorgeaufwendungen und sonstige Vorsorgeaufwendungen) ergeben. Werden auch Beiträge zugunsten eines Basisrentenvertrages (Basisrentenvertrag-Altersvorsorge oder -Erwerbsminderung) geleistet, wird zudem nach einer weiteren Sonderregelung ein Abzugsvolumen ermittelt (Abzugsvolumen nach dem Recht bis 2004 zzgl. Sonderregelung für Basisrentenvertrag). Der höhere Betrag wird abgezogen. In diese Vergleichsberechnung sind nur Vorsorgeaufwendungen einzubeziehen, die nach aktuellem Recht berücksichtigt werden können. Beiträge für eine nach dem 31. Dezember 2004 beginnende Kapitallebensversicherung können beispielsweise auch im Rahmen der Günstigerprüfung nicht angesetzt werden.

\subsection{Riesterförderung}

\subsection{1 „Riester-Rente”}

Eine andere Form der Altersvorsorge ist die sog. "Riester-Rente". Diese wird mit finanziellen Zuschüssen (Altersvorsorgezulagen) und gegebenenfalls mit zusätzlichem Sonderausgabenabzug gefördert. Die Riester-Förderung steht grundsätzlich jedem zu, der wirtschaftlich von den leistungsrechtlichen Auswirkungen der Renten- beziehungsweise Versorgungsreform 2001 betroffen ist und der einem dieser Alterssicherungssysteme weiterhin "aktiv" angehört. Die Förderung soll helfen, diese leistungsrechtlichen Einschnitte auszugleichen. Zur Auswahl stehen verschiedene Anlageformen. Als zertifizierte Vorsorgeverträge können spezielle Rentenversicherungen, Fonds- oder Banksparpläne, Bausparverträge oder Immobiliendarlehen abgeschlossen werden. Im Rahmen dieser Altersvorsorgeverträge kann auch das Risiko einer verminderten Erwerbsfähigkeit abgesichert oder eine Hinterbliebenenversorgung vereinbart werden. Durch die Zertifizierung ist beispielsweise sichergestellt, dass die Altersleistungen lebenslang gezahlt werden und der Anbieter sich verpflichtet, die eingezahlten Beiträge und Zulagen - ohne Abzug von Kosten - für die Altersvorsorge bereitzustellen. Leistungen, die auf geförderten Beiträgen basieren, werden in der Auszahlungsphase besteuert (sog. nachgelagerte Besteuerung).

Wird für die eingezahlten Altersvorsorgebeiträge auf Antrag die volle Altersvorsorgezulage gewährt, beträgt diese ab dem Beitragsjahr 2018 jährlich 175 Euro. Kindergeldempfänger erhalten für jedes Kind zusätzlich 185 Euro im Jahr. Für alle ab 2008 geborenen Kinder erhöht sich diese Kinderzulage auf 300 Euro. Wer vor Beginn des 25. Lebensjahres mit einem Riester-Vertrag vorsorgt, kann einmalig einen Bonus von 200 Euro erhalten (sog. Berufseinsteigerbonus).

Der Zulageantrag ist jährlich bei dem Anbieter des Vertrages (z.B. Versicherungsgesellschaft, Bank, Fondsgesellschaft) zu stellen. Die Antragsfrist beträgt 2 Jahre. Es besteht auch die Möglichkeit, den Anbieter für die Beantragung der Zulage für jedes Beitragsjahr schriftlich zu bevollmächtigen (sog. „Dauerzulagenantrag"). Der Anbieter übermittelt die erforderlichen Vertragsdaten an die Zentrale Zulagenstelle für Altersvermögen (ZfA). Die ZfA errechnet daraufhin den Anspruch auf eine Altersvorsorgezulage und überweist diese an den Anbieter. Der Anbieter schreibt die Zulage dem begünstigten Altersvorsorgevertrag gut.

Darüber hinaus können die geleisteten Altersvorsorgebeiträge zuzüglich der zustehenden Zulage in der Einkommensteuererklärung als Sonderausgaben geltend gemacht werden. Der Sonderausgabenabzug für Altersvorsorgeleistungen beträgt max. 2.100 Euro/Jahr. Dabei prüft 
das Finanzamt, ob sich der Sonderausgabenabzug günstiger auswirkt als der Anspruch auf die Altersvorsorgezulage. Ergibt sich bei der Berechnung durch den Sonderausgabenabzug ein zusätzlicher Steuervorteil, wird dieser im Rahmen der Einkommensteuerveranlagung erstattet.

Die Günstigerprüfung wird durch das Finanzamt von Amts wegen durchgeführt, wenn der Einkommensteuererklärung die Anlage AV beigefügt wird und gegenüber dem Anbieter schriftlich in die elektronische Datenübermittlung eingewilligt wurde. Keine gesonderte Einwilligung in die Datenübermittlung ist erforderlich, wenn beim Anbieter ein Dauerzulagenantrag vorliegt. Außerdem gilt die Einwilligung auch in den Fällen ohne gesonderte Erklärung als erteilt, in denen der mittelbar zulageberechtigte Ehegatte/Lebenspartner einen Zulageantrag beim Anbieter gestellt hat. Der Anbieter unterrichtet jährlich über seine Datenermittlung.

Zum begünstigten Personenkreis gehören insbesondere die Pflichtversicherten in der gesetzlichen Rentenversicherung und der Alterssicherung der Landwirte sowie Beamte, Richter und Soldaten, die im aktiven Dienstverhältnis stehen, oder zeitweise beurlaubte Beamte, deren Beurlaubungszeit ruhegehaltfähig ist bzw. die sich in Elternzeit (Kindererziehungszeit) befinden. Ehegatten/Lebenspartner von begünstigten Personen zählen ebenfalls dazu. Auch geringfügig Beschäftigte können die Förderung erhalten, wenn sie auf die Sozialversicherungsfreiheit verzichten und Rentenversicherungsbeiträge entrichten.

\subsection{2 "Wohn-Riester"}

Für viele stellt das mietfreie Wohnen im Alter eine Art der individuellen Altersvorsorge dar. Sie erhalten mit "Wohn-Riester" (auch
Eigenheimrente genannt) die Möglichkeit, Anschaffung, Bau oder den Barriere reduzierenden Umbau der eigenen Immobilie mit einem privaten Riester-Vertrag (z. B. einem zertifizierten Bausparvertrag oder Immobiliendarlehen) zu finanzieren. Begünstigt ist die selbst genutzte Wohnung, die selbst genutzte Wohnung im eigenen Haus oder das selbst genutzte Haus das sich innerhalb der Europäischen Union oder in einem Staat des Europäischen Wirtschaftsraums (Island, Liechtenstein und Norwegen) befindet.

Zudem besteht die Möglichkeit, das in einem privaten Riester-Vertrag aufgebaute Altersvorsorgevermögen jederzeit ganz oder teilweise für den Aufbau von selbst genutztem Wohneigentum einzusetzen (Altersvorsorge-Eigenheimbetrag). Begünstigt ist die Verwendung des Kapitals für:

Aufwendungen, die in einem unmittelbaren zeitlichen Zusammenhang mit der Anschaffung oder dem Bau einer selbst genutzten, begünstigten Wohnung entstehen.

die Tilgung eines Darlehens, welches für die Anschaffung oder Herstellung einer begünstigten Wohnung aufgenommen wurde. Die Tilgung muss in einem unmittelbaren zeitlichen Zusammenhang mit der Entnahme des angesparten, geförderten Kapitals erfolgen.

Aufwendungen, die in einem unmittelbaren zeitlichen Zusammenhang mit dem Erwerb von Pflichtanteilen an einer eingetragenen Genossenschaft für die Selbstnutzung einer Genossenschaftswohnung entstehen.

die Tilgung eines Darlehens, welches für den Erwerb von Pflichtanteilen an einer eingetragenen Genossenschaft aufgenommen wurde. Die Tilgung muss in einem unmittelbaren zeitlichen Zusammenhang zur Entnahme des angesparten, geförderten Kapitals erfolgen.

den Umbau einer selbstgenutzten und begünstigten Wohnung, sofern dieser 
Umbau zur Reduzierung von Barrieren in und an der begünstigten Wohnung führt.

Die Anschaffung eines eigentumsähnlichen oder lebenslangen Dauerwohnrechts nach § 33 Wohnungseigentumsgesetz steht der Anschaffung einer begünstigen Wohnung gleich.

Die Ausführungen unter 1.3.1 zum begünstigten Personenkreis sowie zur Systematik der Förderung - Gewährung von Zulage oder Sonderausgabenabzug in der Ansparphase - gelten auch für "Wohn-Riester".

Das im Wohneigentum gebundene steuerlich geförderte Altersvorsorgekapital wird - wie bei den anderen Riester-Produkten - nachgelagert besteuert. Zu diesem Zweck werden die geförderten Leistungen und die darauf gewährten Zulagen vertragsbezogen in einem sog. Wohnförderkonto erfasst. Bis zur Auszahlungsphase erhöht sich das Wohnförderkonto jährlich um 2 Prozent. Bei fortdauernder Selbstnutzung der geförderten Wohnung wird das Wohnförderkonto im Rentenalter - wahlweise einmalig mit einem Abschlag oder sukzessive bis zum 85. Lebensjahr - aufgelöst und der Besteuerung unterworfen.

Wird die Selbstnutzung der geförderten Immobilie aufgegeben, kann das geförderte Altersvorsorgekapital für eine andere selbst genutzte Immobilie verwendet werden. Gleichfalls ist es möglich einen Betrag in Höhe des Wohnförderkontos auf einen anderen eigenen RiesterVertrag einzuzahlen. Besondere Regelungen bestehen auch bei beruflich bedingtem Umzug oder soweit die Wohnung krankheits- oder pflegebedingt nicht mehr bewohnt wird. Bei schädlicher Verwendung sind die erhaltenen Zulagen und Steuervorteile zurückzuzahlen.

\subsection{Unterhaltsleistungen an den} geschiedenen oder dauernd getrennt lebenden Ehegatten

Unterhaltsleistungen an den geschiedenen oder dauernd getrennt lebenden Ehegatten können bis zu einem Höchstbetrag von 13.805 Euro jährlich als Sonderausgaben abgezogen werden (sog. Realsplitting). Dieser Betrag erhöht sich um die aufgewandten Beiträge für die Basiskrankenund gesetzliche Pflegeversicherung. Als Unterhaltsleistungen kommen dabei alle Zuwendungen, die ohne Gegenleistung gewährt werden, in Betracht. Unerheblich ist, ob es sich um laufende oder einmalige Leistungen handelt, ob sie in Geld oder Geldeswert (Sachleistungen) bestehen und ob sie freiwillig oder auf Grund der gesetzlichen Unterhaltspflicht geleistet werden. Gleiches gilt für Unterhaltsleistungen bei Aufhebung einer Lebenspartnerschaft oder bei Getrenntlebenden nach dem Lebenspartnerschaftsgesetz.

Die Voraussetzungen für den Abzug als Sonderausgaben sind:

1. Der Unterhaltsleistende muss den Sonderausgabenabzug beim Finanzamt mit Zustimmung des Empfängers beantragen. Der Antrag gilt nur für ein Kalenderjahr und kann nicht zurückgenommen werden. Für den Antrag ist der Vordruck Anlage $U$ zu verwenden, der vom Unterhaltsleistenden unter Angabe der steuerlichen Identifikationsnummer der unterhaltenden Person unterschrieben werden muss. Zusätzlich ist eine Unterschrift des Unterhaltsempfängers erforderlich, sofern seine Zustimmung dem Finanzamt noch nicht vorliegt. Der Unterhaltsempfänger hat die Möglichkeit, die Zustimmung zum Abzug der Unterhaltsleistungen als Sonderausgaben auf einen Teilbetrag zu begrenzen. 
Der Unterhaltsempfänger hat die als Sonderausgaben abgezogenen Zahlungen in der gleichen Höhe als sonstige Einkünfte zu versteuern ( $\rightarrow$ Info-Broschüre "Rund um die Einkommensteuererklärung"). Die Zustimmung ist erstmals für das auf der Anlage $U$ eingetragene Kalenderjahr gültig. Sie gilt solange sie nicht widerrufen wird - auch für darauf folgende Kalenderjahre. Ein Widerruf ist vor Beginn des Kalenderjahrs, für das die Zustimmung erstmals nicht gelten soll, gegenüber dem Wohnsitzfinanzamt des Unterhaltsleistenden oder des Unterhaltsempfängers zu erklären.

2. Der unterstützte Ehegatte/Lebenspartner muss unbeschränkt einkommensteuerpflichtig sein, d.h. dass der Wohnsitz oder gewöhnliche Aufenthalt in der Regel im Inland sein muss. Lebt der Unterhaltsempfänger in einem anderen Mitgliedstaat der Europäischen Union oder einem Staat des Europäischen Wirtschaftsraums (Island, Liechtenstein und Norwegen), sind die Aufwendungen abziehbar, wenn durch eine Bescheinigung der zuständigen ausländischen Steuerbehörde nachgewiesen wird, dass die empfangenen Unterhaltszahlungen tatsächlich besteuert werden. Darüber hinaus existieren entsprechende Regelungen in den Doppelbesteuerungsabkommen mit Dänemark, Kanada, den USA sowie in der mit der Schweiz getroffenen Verständigungsvereinbarung.

3. In den angegebenen Beträgen dürfen keine Unterhaltsleistungen an Kinder enthalten sein.

Übersteigen die Unterhaltsleistungen den Höchstbetrag oder wird der Antrag auf Sonderausgabenabzug auf einen niedrigeren Betrag beschränkt, so kann der nicht als
Sonderausgaben abziehbare Teil der Unterhaltsleistungen auch nicht als außergewöhnliche Belastung $(\rightarrow$ Nr. 2.4.1) berücksichtigt werden.

\subsection{Kirchensteuer}

Als Sonderausgaben abziehbare Kirchensteuern (dazu zählt auch das Kirchgeld) sind Geldleistungen, die als Körperschaften des öffentlichen Rechts anerkannte Religionsgemeinschaften von ihren Mitgliedern auf Grund gesetzlicher Vorschriften erheben.

Abzugsfähig ist die im Kalenderjahr geleistete Kirchensteuer abzüglich der in demselben Kalenderjahr erstatteten Kirchensteuer. Dabei ist es grundsätzlich unerheblich, für welche Jahre die Kirchensteuer geleistet oder erstattet wurde. Kirchensteuern, die auf die sog. Abgeltungsteuer für private Kapitaleinnahmen erhoben werden, sind nicht abziehbar.

Keine Kirchensteuern sind freiwillige Beiträge, die an öffentlich-rechtliche Religionsgemeinschaften geleistet werden. Diese können gegebenenfalls als Spenden geltend gemacht werden $(\rightarrow$ Nr. 1.9).

\subsection{Kinderbetreuungskosten}

Kinderbetreuungskosten sind in Höhe von zwei Drittel der Aufwendungen, höchstens 4.000 Euro je Kind, als Sonderausgaben abziehbar.

Kinderbetreuungskosten werden berücksichtigt, wenn

das Kind unter 14 Jahre alt ist oder wegen einer vor Vollendung des 25. Lebensjahres eingetretenen Behinderung außerstande ist, sich selbst zu unterhalten,

das Kind zum Haushalt des Steuerpflichtigen gehört, 
der Steuerpflichtige für die Aufwendungen eine Rechnung erhalten und

er auf das Konto des Dienstleisters gezahlt hat.

Zu den Kinderbetreuungskosten zählen z. B. Aufwendungen für die Betreuung im Kindergarten, in einer Kindertagesstätte, einer Kinderkrippe oder bei einer Tagesmutter oder Ausgaben für die Beschäftigung einer Haushaltshilfe, soweit sie das Kind betreut. Nicht anerkannt werden Ausgaben für jede Art von Unterricht lauch Nachhilfeunterricht), für die Vermittlung besonderer Fähigkeiten (z. B. Schreibmaschinen- oder Computerkurs) und für sportliche und andere Freizeitaktivitäten sowie für Verpflegung.

Die Rechnung bzw. ein entsprechendes Dokument (z.B. Bescheinigung des Kindergartens über die gezahlten Gebühren, schriftlicher Vertrag) sowie die Zahlungsnachweise sind auf Verlangen des Finanzamts vorzulegen. Barzahlungen werden nicht anerkannt.

Ausführliche Informationen zur Geltendmachung von Kinderbetreuungskosten finden Sie in der $\rightarrow$ Info-Broschüre "Kinder im Steuerrecht".

\subsection{Aufwendungen für die eigene Berufsausbildung}

Aufwendungen für die eigene Berufsausbildung sind bis zu einem Höchstbetrag von 6.000 Euro im Kalenderjahr als Sonderausgaben abziehbar, wenn sie nicht ausnahmsweise Werbungskosten oder Betriebsausgaben sind. Für Ehegatten/ Lebenspartner, die zusammen zur Einkommensteuer veranlagt werden, gilt der vorgenannte Höchstbetrag für jeden Ehegatten/Lebenspartner gesondert. Zu den Aufwendungen für die eigene Berufsausbildung gehören Aufwendungen für eine berufliche Ausbildungsmaßnahme, die die notwendigen fachlichen Kenntnisse und
Fertigkeiten zur erstmaligen Aufnahme eines Berufes vermittelt (Erstausbildung = erstmalige Berufsausbildung oder Erststudium als Erstausbildung).

Eine erstmalige Berufsausbildung liegt vor, wenn es sich um eine geordnete Ausbildung mit einer Mindestdauer von 12 Monaten bei vollzeitiger Ausbildung und mit einer Abschlussprüfung handelt. Eine geordnete Ausbildung ist anzunehmen, wenn sie auf der Grundlage von Rechts- oder Verwaltungsvorschriften oder internen Vorschriften eines Bildungsträgers durchgeführt wird. Bedarf es keiner Abschlussprüfung, muss die Ausbildung zumindest tatsächlich planmäßig beendet werden. Eine abgebrochene Berufsausbildung erfüllt diese Bedingung nicht. Bedarf es nicht des Durchlaufens einer Berufsausbildung, gilt das erfolgreiche Absolvieren einer Abschlussprüfung als abgeschlossene Erstausbildung.

Ein Erststudium als Erstausbildung liegt vor, wenn zuvor keine erste Berufsausbildung abgeschlossen wurde. Um ein Studium handelt es sich, wenn es an einer Hochschule im Sinne der Hochschulgesetze der Länder (z.B. Universität, Pädagogische Hochschule, Kunsthochschule, Fachhochschule) absolviert wird. Das Studium kann auch als Fernstudium durchgeführt werden.

Als Sonderausgaben abzugsfähig sind u.a.:

Studiengebühren

Kosten für Lehrbücher und andere Arbeitsmittel

- Aufwendungen für Wege zwischen Wohnung und Ausbildungsort in Höhe der Entfernungspauschale

- Mehraufwendungen wegen doppelter Haushaltsführung und

- Fahrtkosten und Verpflegungsmehraufwendungen für gelegentliche Reisen zu anderen 
Ausbildungsorten (hier gelten Dienstreisegrundsätze, siehe $\rightarrow$ Anleitung zur Einkommensteuererklärung, Anlage $\mathrm{N}$ - Reisekosten bei beruflich veranlasster Auswärtstätigkeit/ Fahrt- und Übernachtungskosten, Reisenebenkosten).

Steuerfreie Bezüge, die ausschließlich zur Bestreitung der Berufsausbildungskosten gezahlt werden, mindern die abziehbaren Aufwendungen entsprechend. Eine Kürzung unterbleibt, wenn die steuerfreien Bezüge ausschließlich oder teilweise Aufwendungen für den Lebensunterhalt abgelten (z. B. Berufsausbildungsbeihilfen nach $\S 59$ SGB III, Leistungen nach den $\S \S 12$ und 13 BAföG); dies gilt jedoch nicht bei Leistungen für auswärtige Unterbringung.

Steuerlich wirkt sich der Abzug der o. g. Aufwendungen nur aus, wenn eigene Einkünfte vorliegen. Hat z. B. ein Student oder Auszubildender in dem Jahr, in dem die Ausgaben angefallen sind, keine eigenen Einkünfte, kann ein nicht ausgenutzter Sonderausgabenabzug nicht auf spätere Jahre vorgetragen werden.

Findet die Ausbildung im Rahmen eines Ausbildungsdienstverhältnisses statt, sind die in diesem Zusammenhang anfallenden Kosten nicht als Sonderausgaben, sondern als Werbungskosten zu berücksichtigen. Dies gilt auch für Aufwendungen für Fortbildungen in einem bereits erlernten Beruf und für Umschulungsmaßnahmen zur Vorbereitung eines Berufswechsels oder für ein weiteres Studium nach Abschluss des Erststudiums. Voraussetzung für den Werbungskostenabzug (oder Betriebsausgabenabzug) ist, dass die Aufwendungen in einem konkreten, objektiv feststellbaren Zusammenhang mit späteren im Inland steuerpflichtigen Einnahmen aus der angestrebten beruflichen Tätigkeit stehen.
Praktika und Anerkennungsjahre sind Bestandteil der erstmaligen Berufsausbildung, soweit keine vorherige abgeschlossene Berufsausbildung vorangegangen ist. Kosten für ein nach einer abgeschlossenen Berufsausbildung oder nach einem berufsqualifizierenden Studium aufgenommenes Praktikum können dagegen Werbungskosten sein.

\subsection{Schulgeld}

Als Sonderausgaben können 30\% des Entgelts, höchstens 5.000 Euro, für den Besuch eines Kindes in einer Schule in freier Trägerschaft oder einer überwiegend privat finanzierten Schule abgezogen werden. Entgelte für Beherbergung, Betreuung und Verpflegung werden nicht berücksichtigt. Der Höchstbetrag wird für jedes Kind je Elternpaar nur einmal gewährt.

Voraussetzung ist, dass die Schule in einem Mitgliedstaat der Europäischen Union oder des Europäischen Wirtschaftsraumes (Island, Liechtenstein und Norwegen) belegen ist. Ferner muss die Schule zu einem vom zuständigen inländischen Ministerium eines Landes (in Sachsen: Sächsisches Staatsministerium für Kultus), von der Kultusministerkonferenz der Länder oder von einer inländischen Zeugnisanerkennungsstelle anerkannten Abschluss führen oder einem inländischen Abschluss an einer öffentlichen Schule gleichwertig sein. Der Besuch einer Deutschen Schule im Ausland steht dem Besuch einer Schule im o.g. Sinne - unabhängig von ihrer Belegenheit - gleich.

Weitere Voraussetzung ist, dass für das Kind ein Anspruch auf Freibeträge für Kinder oder Kindergeld besteht $(\rightarrow$ Info-Broschüre "Kinder im Steuerrecht"). 


\subsection{Spenden}

Zuwendungen (Spenden und Mitgliedsbeiträge) zur Förderung kirchlicher, mildtätiger und gemeinnütziger Zwecke sind bis zur Höhe von $20 \%$ des Gesamtbetrages der Einkünfte als Sonderausgaben abziehbar. Alternativ dazu können Unternehmer Spenden bis zu einer Höhe von 4 von Tausend der Summe ihres Jahresumsatzes und der im Kalenderjahr aufgewendeten Löhne und Gehälter abziehen. Zuwendungen, die diese Höchstbeträge übersteigen, werden auf folgende Veranlagungszeiträume übertragen (Spendenvortrag).

Begünstigt sind neben Geld- auch Sachzuwendungen.

Dagegen sind Mitgliedsbeiträge an Vereine, deren Tätigkeit in erster Linie der Freizeitgestaltung ihrer Mitglieder dient, z.B. Sport-, Heimat- oder Gesangsvereine, steuerlich nicht begünstigt.

Darüber hinaus können Spenden in das zu erhaltende Vermögen (Vermögensstock) von Stiftungen bis zu einem Gesamtbetrag von 1 Million Euro im Jahr der Zuwendung und in den folgenden 9 Kalenderjahren als Sonderausgaben abgezogen werden. Bei Zusammenveranlagung erhöht sich der Gesamtbetrag auf 2 Millionen Euro. Unerheblich ist, ob die Spende aus dem gemeinsamen Vermögen oder aus dem Vermögen eines Ehegatten/Lebenspartners geleistet wird.

Für Mitgliedsbeiträge und Spenden an politische Parteien sieht das Einkommensteuergesetz eine Steuerermäßigung nach $\S 34 \mathrm{~g}$ EStG vor. Die tarifliche Einkommensteuer vermindert sich um $50 \%$ der im Kalenderjahr geleisteten Mitgliedsbeiträge und Spenden, maximal 825 Euro bzw. 1.650 Euro bei Zusammenveranlagung. Dieser
Steuerermäßigungsbetrag verringert die Einkommensteuer höchstens auf null Euro.

Übersteigen die im Kalenderjahr geleisteten Mitgliedsbeiträge und Spenden an politische Parteien 1.650 Euro bzw. bei Zusammenveranlagung 3.300 Euro, kann der übersteigende Betrag als Sonderausgabe abgezogen werden. Der Sonderausgabenabzug ist auf 1.650 Euro, bei Zusammenveranlagung auf 3.300 Euro begrenzt. Höhere Zuwendungen an politische Parteien wirken sich steuerlich nicht aus.

\section{Beispiel:}

Herr Meier, ledig, hat im Jahr 2018 Spenden an politische Parteien von insgesamt 4.000 Euro geleistet:

Steuerermäßigung nach $\S 34 \mathrm{~g}$ EStG

$50 \%$ der Aufwendungen, max.

825 Euro

Sonderausgabenabzug für die verblei-

bende Spende (4.000 Euro - 1.650 Euro =) 2.350 Euro

davon max. abziehbar sind

1.650 Euro

(Höchstbetrag)

Zuwendungen für steuerbegünstigte Zwecke sind grundsätzlich durch eine Zuwendungsbestätigung nach amtlich vorgeschriebenem Vordruck nachzuweisen.

Bei Zuwendungen bis zu 200 Euro genügt als vereinfachter Nachweis der Bareinzahlungsbeleg oder die Buchungsbestätigung des Kreditinstituts (z. B. Kontoauszug), wenn der Zuwendungsempfänger:

【 eine inländische juristische Person des öffentlichen Rechts oder eine inländische öffentliche Dienststelle ist oder

eine als gemeinnützig anerkannte Körperschaft (Verein, Stiftung) ist und der steuerbegünstigte Zweck und die Angaben über die Freistellung von der Körperschaftsteuer auf einem von ihr hergestellten Beleg aufgedruckt sind und darauf angegeben ist, ob es 
sich um eine Spende oder einen Mitgliedsbeitrag handelt oder

eine politische Partei im Sinne des § 2 Parteiengesetz ist und bei Spenden der Verwendungszweck auf dem von ihr hergestellten Beleg aufgedruckt ist.

Als Nachweis für die Zahlung von Mitgliedsbeiträgen an politische Parteien genügen Bareinzahlungsbelege, Buchungsbestätigungen oder Beitragsquittungen.

Für Spenden zur Hilfe in Katastrophenfällen, die innerhalb eines bestimmten Zeitraumes auf ein speziell eingerichtetes Sonderkonto einer inländischen juristischen Person des öffentlichen Rechts, einer inländischen öffentlichen Dienststelle oder eines inländischen amtlich anerkannten Verbandes der freien Wohlfahrtspflege (z.B. Deutsches Rotes Kreuz e.V. oder Deutscher Caritasverband e.V.) eingezahlt wurden, gilt ohne betragsmäßige Beschränkung ebenfalls der vereinfachte Zuwendungsnachweis. Dieser genügt auch, wenn die Spenden bereits vor Einrichtung eines Sonderkontos auf ein anderes Konto der oben genannten Zuwendungsempfänger geleistet werden. Bei Zuwendungen in Katastrophenfällen, die über ein Konto eines Dritten geleistet werden, gelten besondere Regelungen.

\subsection{Sonderausgaben-Pauschbetrag/ Vorsorgepauschale}

Werden keine höheren Aufwendungen nachgewiesen, wird u.a. für die unter Nr. 1.4 bis Nr. 1.9 angeführten Sonderausgaben ein jährlicher Pauschbetrag von 36 Euro bzw. 72 Euro bei Zusammenveranlagung berücksichtigt.

Vorsorgeaufwendungen $(\rightarrow$ Nr. 1.2) werden im Rahmen der Einkommensteuerveranlagung nur in tatsächlicher Höhe berücksichtigt. Der Ansatz einer Pauschale ist hierfür bei der Einkommensteuerveranlagung nicht möglich.

Im Lohnsteuerabzugsverfahren wird für Altersvorsorge- und sonstige Vorsorgeaufwendungen $(\rightarrow$ Nr. 1.2.1 und 1.2.2) dagegen eine Vorsorgepauschale abgezogen, die sich aus folgenden Teilbeträgen zusammensetzt:

- Teilbetrag für die Rentenversicherung,

- Teilbetrag für die Krankenversicherung und

Teilbetrag für die Pflegeversicherung.

Darüber hinaus ist die Berücksichtigung weiterer Vorsorgeaufwendungen als Freibetrag beim Lohnsteuerabzug nicht möglich.

Vereinfacht dargestellt, entspricht die Vorsorgepauschale bei sozialversicherungspflichtigen Arbeitnehmern grundsätzlich der Summe aus den Kranken- und sozialen Pflegeversicherungsbeiträgen (Arbeitnehmeranteil ohne Beitragsteile für das Krankengeld) sowie dem abziehbaren Teil der Rentenversicherungsbeiträge.

Privat versicherte Arbeitnehmer können ihrem Arbeitgeber die Höhe der Beträge zur Basiskranken- und Pflege-Pflichtversicherung (auch für ihre Kinder bzw. den nicht erwerbstätigen Ehegatten/Lebenspartner) durch Vorlage einer entsprechenden Beitragsbescheinigung nachweisen. Dem Arbeitgeber bereits vorgelegte Bescheinigungen über die voraussichtlichen privaten Basiskranken- und Pflege-Pflichtversicherungsbeiträge eines Kalenderjahres sind grundsätzlich weiter zu berücksichtigen, wenn keine neue Beitragsmitteilung des Versicherungsunternehmens vorliegt.

Die Teilbeträge für die Krankenversicherung und die gesetzliche Pflegeversicherung sind in der Summe mindestens in Höhe der sog. 
Mindestvorsorgepauschale anzusetzen. Die Ist die beim Lohnsteuerabzug berücksichtigte Mindestvorsorgepauschale beträgt $12 \%$ des Vorsorgepauschale höher als die Summe der Arbeitslohns, höchstens 3.000 Euro in Steuerklasse III und in den anderen Steuerklassen 1.900 Euro jährlich. als Sonderausgaben abziehbaren tatsächlichen Vorsorgeaufwendungen, besteht eine Pflicht zur Abgabe der Einkommensteuererklärung. 


\section{Außergewöhnliche Belastungen}

\subsection{Was sind außergewöhnliche Belastungen?}

Außergewöhnliche Belastungen sind Kosten der privaten Lebensführung, die auf Grund besonderer Umstände anfallen und nur auf Antrag in einem bestimmten Umfang steuerlich berücksichtigt werden. Sie müssen zwangsläufig entstehen und außergewöhnlich sein.

Aufwendungen erwachsen einem Steuerpflichtigen dann zwangsläufig, wenn er sich ihnen aus rechtlichen, tatsächlichen oder sittlichen Gründen nicht entziehen kann. Deshalb können in der Regel nur Aufwendungen des Steuerpflichtigen für sich selbst oder für Angehörige anerkannt werden. Zudem müssen die Kosten den Umständen nach notwendig sein und dürfen einen angemessenen Betrag nicht übersteigen.

Außergewöhnlich sind Aufwendungen, wenn sie höher sind als bei der überwiegenden Mehrzahl der Steuerpflichtigen gleicher Einkommens- und Vermögensverhältnisse und gleichen Familienstandes.

Die außergewöhnlichen Belastungen sind im Einkommensteuergesetz in den $\S \S 33$ bis 336 geregelt. Dort wird unterschieden zwischen außergewöhnlichen Belastungen allgemeiner Art und außergewöhnlichen Belastungen in besonderen Fällen. In der Einkommensteuererklärung sind die Aufwendungen im Mantelbogen, Seite 3 bzw. - soweit sie Kinder betreffen - auf der Anlage Kind zu erklären. Für die Geltendmachung von Unterhaltsaufwendungen ist die Anlage Unterhalt zu verwenden.

Abzugszeitpunkt ist das Kalenderjahr, in dem die Ausgaben tatsächlich geleistet worden sind. Ersatzleistungen Dritter mindern die abziehbaren Aufwendungen. Dies gilt auch dann, wenn diese erst in einem späteren Kalenderjahr gezahlt werden, der Steuerpflichtige aber bereits in dem Kalenderjahr, in dem die Belastung eingetreten ist, mit der Zahlung rechnen konnte. In diesem Fall können nur die geleisteten Aufwendungen, gekürzt um die Ersatzleistung, abgezogen werden.

\subsection{Außergewöhnliche Belastungen allgemeiner Art}

$\mathrm{Zu}$ den außergewöhnlichen Belastungen allgemeiner Art zählen Ausgaben, die z.B. durch Krankheit, Todesfall, Unwetterschäden oder wegen Behinderung entstanden sind. Eine Steuerermäßigung kommt nur in Betracht, wenn die Aufwendungen einen bestimmten Teil des Einkommens übersteigen (sog. zumutbare Belastung ( $\rightarrow$ Nr. 2.3). Ein Ausgabenersatz von dritter Seite (z.B. Versicherungsleistungen, Beihilfen, Unterstützungen, Entschädigungen) vermindert die berücksichtigungsfähigen Aufwendungen, da der Steuerpflichtige insoweit nicht endgültig belastet ist. Die Aufwendungen sind grundsätzlich im Einzelnen nachzuweisen bzw. glaubhaft zu machen. Behinderte Menschen und Personen, denen laufende Hinterbliebenenbezüge 
bewilligt worden sind, sowie Steuerpflichtige, die eine ständig hilflose Person pflegen, können aber auch einen Pauschbetrag geltend machen (Behinderten-, Hinterbliebenen- und PflegePauschbetrag). Diese Pauschbeträge werden nicht um die zumutbare Belastung gekürzt.

Nachfolgend werden einzelne außergewöhnliche Belastungen näher erläutert:

\subsubsection{Krankheit}

Krankheitskosten sind Aufwendungen, die zum Zweck der Heilung einer Krankheit oder mit dem Ziel getätigt werden, die Krankheit erträglicher zu machen bzw. die Krankheitsfolgen zu lindern. Zu den Krankheitskosten gehören beispielsweise Behandlungskosten eines Arztes oder zugelassenen Heilpraktikers, Krankenhauskosten sowie Aufwendungen für verordnete Arznei-, Heil- und Hilfsmittel (z.B. Hör- und Sehhilfen, orthopädische Einlagen, Zahnersatz).

Nicht abziehbar sind z.B. Kosten für medizinische Fachliteratur, für Diätverpflegung oder Trinkgelder. Rein altersbedingte Mehraufwendungen sind grundsätzlich ebenfalls nicht - insbesondere nicht als Krankheitskosten - abziehbar, da diese Aufwendungen nicht außergewöhnlich sind.

Die medizinische Notwendigkeit der Aufwendungen für Arznei-, Heil- und Hilfsmittel ist dem Finanzamt durch Verordnung eines Arztes oder Heilpraktikers nachzuweisen. Bei einer andauernden Erkrankung mit anhaltendem Verbrauch bestimmter Arznei-, Heil- und Hilfsmittel genügt die einmalige Vorlage einer Verordnung. Wurde die Notwendigkeit einer Sehhilfe in der Vergangenheit durch einen Augenarzt festgestellt, genügt in den Folgejahren die Sehschärfenbestimmung durch einen Augenoptiker.
Für bestimmte Ausgaben ist als Nachweis der Zwangsläufigkeit und Notwendigkeit der Aufwendungen ein vor dem Kauf oder der Behandlung ausgestelltes amtsärztliches Attest vorzulegen, z. B. für

Kuren

psychotherapeutische Behandlungen

die medizinisch erforderliche auswärtige Unterbringung eines an einer Behinderung leidenden Kindes

die Notwendigkeit der Betreuung durch eine Begleitperson, sofern sich diese nicht bereits aus dem Ausweis nach § 152 SGB IX (Schwerbehindertenausweis) ergibt

medizinische Hilfsmittel, die als allgemeine Gebrauchsgegenstände des täglichen Lebens anzusehen sind

wissenschaftlich nicht anerkannte Behandlungsmethoden, wie Frisch- und Trockenzellenbehandlungen, Sauerstoff-, Chelat- und Eigenbluttherapie.

Dem amtsärztlichen Attest gleichgestellt sind eine ärztliche Bescheinigung eines Medizinischen Dienstes der Krankenversicherung, bei Pflichtversicherten die Bescheinigung der Versicherungsanstalt und bei öffentlichen Bediensteten die Bescheinigung über die Anerkennung der Beihilfefähigkeit durch die Beihilfestellen.

Fahrtkosten sind grundsätzlich weder außergewöhnliche noch zwangsläufige Aufwendungen und daher nicht abziehbar. Eine Ausnahme gilt aber z.B. für Fahrten zum Arzt aus Anlass einer Behandlung, für Kosten eines Krankentransports oder bei Kraftfahrzeugkosten behinderter Menschen.

Aufwendungen für unumgängliche Fahrten, welche ausnahmsweise als außergewöhnliche Belastung anerkannt werden können, sind bei Benutzung eines Pkw grundsätzlich nur in Höhe der Kosten abziehbar, die bei Benutzung 
eines öffentlichen Verkehrsmittels entstanden wären, es sei denn, die Fahrt mit öffentlichen Verkehrsmitteln ist auf Grund der ungünstigen Verbindung unzumutbar.

\section{Aufwendungen für die üblichen Besuchsfahrten} zu nahen Angehörigen sind im Regelfall nicht abziehbar. Davon ausgenommen sind Aufwendungen für Besuchsfahrten zu einem über längere Zeit in einem Krankenhaus liegenden Ehegatten/ Lebenspartner oder Kind, wenn der behandelnde Krankenhausarzt durch Attest bescheinigt, dass gerade der Besuch des Steuerpflichtigen zur Linderung oder Heilung einer bestimmten Krankheit entscheidend beitragen kann. Keine außergewöhnliche Belastung sind Aufwendungen für den Besuch eines Angehörigen in der Kur sowie Aufwendungen eines Elternteils für Besuche seiner bei dem anderen Elternteil lebenden Kinder.

\subsubsection{Behinderung}

\section{Tatsächliche Aufwendungen}

Aufwendungen, die einem behinderten Menschen wegen seiner Behinderung entstehen, können als außergewöhnliche Belastung berücksichtigt werden, z. B.

- Ausgaben für erhöhten Wäscheverbrauch und besondere Hilfeleistungen

I Kosten für behinderungsbedingte Heilbehandlungen und Medikamente

Fahrtkosten und

- Aufwendungen für die Beschäftigung einer ambulanten Pflegekraft.

Mehraufwendungen für die notwendige behindertengerechte Gestaltung eines für den eigenen Wohnbedarf eingerichteten Hauses oder einer Wohnung können eine außergewöhnliche Belastung darstellen, soweit die Baumaßnahmen durch die Behinderung bedingt sind (z. B. behindertengerechter Umbau einer Dusche). Die
Zwangsläufigkeit der Aufwendungen ist anhand der folgenden Unterlagen nachzuweisen:

- Bescheid eines gesetzlichen Trägers der Sozialversicherung oder der Sozialleistungen über die Bewilligung eines pflege- bzw. behinderungsbedingten Zuschusses (z. B. zur Verbesserung des individuellen Wohnumfeldes nach $\S 40$ Absatz 4 SGB XI) oder

- Gutachten des Medizinischen Dienstes der Krankenversicherung, des Sozialmedizinischen Dienstes oder der Medicproof Gesellschaft für Medizinische Gutachten mbH oder

- Bescheid der Beihilfestelle oder des Trägers der freien Heilfürsorge.

Die abziehbaren Aufwendungen sind im Jahr der Zahlung zu berücksichtigen; eine Verteilung auf mehrere Veranlagungszeiträume ist nicht zulässig.

Kraftfahrzeugkosten behinderter Menschen, die durch die Behinderung verursacht worden sind, können wie folgt berücksichtigt werden:

Bei geh- und stehbehinderten Menschen mit einem Grad der Behinderung (GdB) von mindestens 80 oder mindestens 70 mit Merkzeichen "G" werden Aufwendungen für unvermeidbare Fahrten in angemessenem Rahmen anerkannt, soweit diese nachgewiesen oder glaubhaft gemacht worden sind. Aus Vereinfachungsgründen können grundsätzlich ohne weitere Einzelnachweise 0,30 Euro/km für Fahrten bis zu 3.000 km jährlich angesetzt werden. Bei außergewöhnlich gehbehinderten $\left({ }^{\prime} \mathrm{aG} \mathrm{G}^{\prime \prime}\right)$, blinden $\left({ } \mathrm{BI}{ }^{\prime \prime}\right)$ oder hilflosen $\left.{ }_{1} \mathrm{H}^{\prime \prime}\right)$ Menschen kann eine Fahrleistung bis zu $15.000 \mathrm{~km}$ berücksichtigt werden, wenn entsprechende tatsächliche Fahrten nachgewiesen oder glaubhaft gemacht wurden.

Behinderte Menschen, die die Voraussetzung für die Gewährung eines Pauschbetrags erfüllen 
( $\rightarrow$ siehe nachfolgende Erläuterungen), haben für jeden Veranlagungszeitraum ein Wahlrecht, ob sie entweder ohne Einzelnachweis den Behinderten-Pauschbetrag oder aber unter Kürzung um die zumutbare Belastung $(\rightarrow$ Nr. 2.3) die tatsächlich entstandenen, höheren behinderungsbedingten Aufwendungen geltend machen. Dafür sind zumindest für einen begrenzten Zeitraum entsprechende Aufzeichnungen zu führen und Belege zu sammeln.

\section{Pauschbetrag für behinderte Menschen}

Wegen der Aufwendungen für die Hilfe bei den gewöhnlichen und regelmäßig wiederkehrenden Verrichtungen des täglichen Lebens, für die Pflege sowie für einen erhöhten Wäschebedarf wird auf Antrag anstelle der tatsächlichen Aufwendungen ein Behinderten-Pauschbetrag gewährt. Dieser wird nicht um die zumutbare Belastung $(\rightarrow$ Nr. 2.3) gekürzt. Der Pauschbetrag ist ein Jahresbetrag, der auch dann in voller Höhe gewährt wird, wenn die Behinderung nicht während des gesamten Jahres vorgelegen hat. Die Höhe des Behinderten-Pauschbetrags ist gestaffelt und richtet sich nach dem dauernden GdB. Bei Änderungen im Laufe eines Kalenderjahres wird stets der Pauschbetrag nach dem höchsten festgestellten GdB gewährt:

\begin{tabular}{|c|c|}
\hline Grad der Behinderung & Pauschbetrag \\
\hline 25 und 30 & 310 Euro \\
\hline 35 und 40 & 430 Euro \\
\hline 45 und 50 & 570 Euro \\
\hline 55 und 60 & 720 Euro \\
\hline 65 und 70 & 890 Euro \\
\hline 75 und 80 & 1.060 Euro \\
\hline 85 und 90 & 1.230 Euro \\
\hline 95 und 100 & 1.420 Euro \\
\hline
\end{tabular}

Bei einem $G d B$ von mindestens 50 wird der Behinderten-Pauschbetrag ohne weitere Voraussetzungen gewährt. Bei einem geringeren GdB kann der Behinderten-Pauschbetrag nur gewährt werden, wenn dem behinderten Menschen wegen seiner Behinderung nach gesetzlichen Vorschriften Renten (z. B. Unfallrente) oder andere laufende Bezüge zustehen. Der Behinderten-Pauschbetrag steht auch zu, wenn durch eine Bescheinigung nachgewiesen wird, dass die Behinderung zu einer dauernden Einbuße der körperlichen Beweglichkeit geführt hat oder auf einer typischen Berufskrankheit beruht. Diese Bescheinigung erteilen im Freistaat Sachsen die Landkreise bzw. Kreisfreien Städte.

Für Blinde (Merkzeichen "BI") und behinderte Menschen, die nicht nur vorübergehend hilflos sind (Merkzeichen "H" bzw. Einstufung in die Pflegegrade 4 und 5 nach dem SGB XI, dem $S G B X I I$ oder diesen entsprechenden Vorschriften), wird unabhängig vom $\mathrm{GdB}$ ein erhöhter Behinderten-Pauschbetrag von 3.700 Euro/Jahr gewährt.

Die Behinderung ist dem Finanzamt durch eine Bescheinigung der nach § 152 SGB IX zuständigen Behörde (im Freistaat Sachsen: der Landkreis bzw. die Kreisfreie Stadt), einen entsprechenden Rentenbescheid (falls ein GdB unter 50 festgestellt worden ist), einen Schwerbehindertenausweis oder einen Bescheid über die Einstufung als Schwerstpflegebedürftiger in die Pflegegrade 4 und 5 (Merkzeichen ${ } \mathrm{H}$ ) nachzuweisen.

Steht einem Kind ein Behinderten-Pauschbetrag zu, für das der Steuerpflichtige Anspruch auf Kindergeld oder einen Freibetrag für Kinder hat, wird der Pauschbetrag auf Antrag auf den Steuerpflichtigen übertragen, wenn das Kind den Pauschbetrag (z.B. mangels eigener Einkünfte) nicht in Anspruch nimmt. Bei Eltern, 
die nicht zusammen veranlagt werden, wird der Pauschbetrag dabei grundsätzlich auf beide Elternteile je zur Hälfte aufgeteilt, es sei denn, der Kinderfreibetrag wurde auf einen Elternteil übertragen. Auf gemeinsamen Antrag der Eltern ist auch eine andere Aufteilung möglich. Nähere Informationen zum Kindergeld oder den Freibeträgen für Kinder erhalten Sie in der $\rightarrow$ InfoBroschüre "Kinder im Steuerrecht".

Mit dem Behinderten-Pauschbetrag werden nur die typischen behinderungsbedingten Mehraufwendungen abgegolten, die unmittelbar infolge der Behinderung entstehen. Neben diesem Pauschbetrag können einmalige, untypische und außerordentliche Kosten (z.B. Aufwendungen für eine Heilkur, Kosten einer Operation) sowie mittelbare behinderungsbedingte Aufwendungen (z.B. Kraftfahrzeugkosten $\rightarrow$ Seite 19) abgezogen werden; die zumutbare Belastung ist anzurechnen $(\rightarrow \mathrm{Nr}$. 2.3). Wird ein Behinderten-Pauschbetrag geltend gemacht, können jedoch die tatsächlichen Ausgaben wegen Pflegebedürftigkeit (z.B. Aufwendungen für eine ambulante Pflegekraft oder für eine Heimunterbringung) nicht zusätzlich als außergewöhnliche Belastung geltend gemacht oder im Rahmen des § 35a EStG - SteuerermäBigung bei Aufwendungen für haushaltsnahe Beschäftigungsverhältnisse und für die Inanspruchnahme haushaltsnaher Dienstleistungen - berücksichtigt werden $(\rightarrow$ Info-Broschüre „Was, Wie, Wo? - Rund um die Einkommensteuererklärung").

Unabhängig von der Übertragung des Behinderten-Pauschbetrags können Eltern bzw. Großeltern ihre eigenen zwangsläufigen Aufwendungen für ein behindertes Kind als außergewöhnliche Belastung allgemeiner Art abziehen (z.B. entstandene Kosten für Fahrten mit dem behinderten Kind im Rahmen der Angemessenheit) oder für übernommene Pflege- und Betreuungsaufwendungen des behinderten Kindes eine Steuerermäßigung nach $\S 35 a \mathrm{EStG}$ in Anspruch nehmen.

\subsubsection{Pflegebedürftigkeit}

\section{Abziehbare Aufwendungen}

Aufwendungen wegen eigener Pflegebedürftigkeit oder für die Pflege eines Angehörigen können als außergewöhnliche Belastung abziehbar sein. Erstattungen von dritter Seite (z. B. Pflegekassen) sind anzurechnen. Die Übernahme der Pflegekosten für Angehörige ist aus rechtlichen Gründen zwangsläufig, wenn der Steuerpflichtige der gepflegten Person gegenüber gesetzlich unterhaltsverpflichtet ist, $d$. h. beispielsweise gegenüber Kindern, Eltern und Großeltern. Anderen Verwandten gegenüber kann eine Zwangsläufigkeit aus tatsächlichen oder sittlichen Gründen vorliegen; allein das Bestehen eines nahen Verwandtschaftsverhältnisses reicht jedoch nicht aus. Hat die zu pflegende Person eigene Einkünfte oder eigenes Vermögen und ist sie daher in der Lage, die Aufwendungen selbst zu tragen, kann es an der Zwangsläufigkeit fehlen.

Zu den Aufwendungen infolge eigener Pflegebedürftigkeit zählen sowohl Kosten für einen ambulanten Pflegedienst, Einrichtungen der Tages-, Nacht- oder Kurzzeitpflege als auch Aufwendungen zur Unterbringung in einem Heim. Wurde der private Haushalt aufgelöst, sind die Kosten einer Heimunterbringung nur insoweit zu berücksichtigen, als sie die sog. Haushaltsersparnis übersteigen. Die Haushaltsersparnis wird in Höhe des Höchstbetrags für 
Unterhaltsleistungen nach § 33a Absatz 1 EStG (2018: 9.000 Euro') geschätzt.

Voraussetzung für die steuerliche Berücksichtigung der Aufwendungen ist, dass beim Steuerpflichtigen selbst bzw. der pflegebedürftigen Person ein Pflegegrad (1 bis 5) im Sinne der $\S \S 14,15$ SGB XI festgestellt wurde. Dies ist durch eine Bescheinigung der sozialen Pflegekasse bzw. eines privaten Versicherungsunternehmens oder - falls das gesundheitliche Merkmal "hilflos" festgestellt wurde - durch den Schwerbehindertenausweis nachzuweisen.

Bei ambulant gepflegten Personen, für die kein Pflegegrad festgestellt wurde, können die Pflegekosten ohne weiteren Nachweis als außergewöhnliche Belastung berücksichtigt werden, wenn sie von einem anerkannten Pflegedienst nach § 89 SGB XI in Rechnung gestellt worden sind.

Die tatsächlichen Ausgaben wegen eigener Pflegebedürftigkeit können dann nicht als außergewöhnliche Belastung geltend gemacht werden, wenn ein Behinderten-Pauschbetrag $(\rightarrow$ Seite 21) in Anspruch genommen wird.

Werden die tatsächlichen Aufwendungen als außergewöhnliche Belastung geltend gemacht, erfolgt von Amts wegen die Kürzung um die zumutbare Belastung $(\rightarrow$ Nr. 2.3).

\section{Pflege-Pauschbetrag}

Ein Steuerpflichtiger kann wegen der außergewöhnlichen Belastungen, die inm durch die persönliche Pflege einer anderen nicht nur

\footnotetext{
1 Nach dem Entwurf des FamEntlastG sind folgende Änderungen geplant:

in 2019: 9.168 Euro

ab 2020: 9.408 Euro
}

vorübergehend hilflosen Person (Merkzeichen "H" im Schwerbehindertenausweis oder Pflegegrade 4 und 5) in seiner oder in deren Wohnung im Inland entstehen, anstelle seiner tatsächlichen Aufwendungen einen PflegePauschbetrag in Höhe von 924 Euro erhalten. Die Pflege wird auch dann noch persönlich vorgenommen, wenn sich der Steuerpflichtige zur Unterstützung zeitweise der Mithilfe einer anderen Person (z.B. ambulante Pflegekraft) bedient. Weitere Voraussetzung ist, dass er für die Pflege keine Einnahmen erhält.

Einnahmen sind z.B. Pflegegelder aus der gesetzlichen oder privaten Pflegeversicherung, die die hilflose Person an den Steuerpflichtigen weitergibt, um seine Pflegedienstleistungen zu vergüten oder seine entstandenen Aufwendungen zu ersetzen. Das Pflegegeld zählt nicht zu den Einnahmen der Pflegeperson, wenn es

- Eltern für ihr behindertes Kind erhalten,

- der Steuerpflichtige lediglich treuhänderisch für den Pflegebedürftigen verwaltet und damit ausschließlich Aufwendungen des Pflegebedürftigen bestreitet; die Pflegeperson muss die konkrete Verwendung des Pflegegeldes nachweisen können.

Beiträge zur Renten-, Kranken- und Pflegeversicherung der Pflegeperson, die die Pflegekasse übernimmt, führen ebenfalls allein nicht zu Einnahmen, die eine Gewährung des PflegePauschbetrags ausschließen.

Der Pflege-Pauschbetrag ist ein Jahresbetrag, der nicht um die zumutbare Belastung gekürzt wird. Er ist nach der Zahl der Pflegepersonen aufzuteilen, wenn ein Pflegebedürftiger während des Kalenderjahres gleichzeitig oder nacheinander von mehreren Personen gepflegt wird. Pflegt ein Steuerpflichtiger mehrere hilflose 
Personen, so steht inm der Pauschbetrag mehrfach zu.

Neben dem Pflege-Pauschbetrag können weitere Aufwendungen, z. B. für eine Heilkur, Operation oder Kraftfahrzeugkosten $(\rightarrow$ Seite 19$)$ sowie der Behinderten-Pauschbetrag $(\rightarrow$ Seite 20) abgezogen werden. Der Pflege-Pauschbetrag kann neben dem auf die Eltern oder Großeltern eines behinderten Kindes übertragenen Behinderten-Pauschbetrags gewährt werden. Für Pflege- und Betreuungsaufwendungen sowie Aufwendungen für eine Hilfe im Haushalt ist außerdem die Steuerermäßigung nach § 35a EStG $(\rightarrow$ Info-Broschüre „Was, Wie, Wo? - Rund um die Einkommensteuererklärung") möglich.

\subsubsection{Hinterbliebenen-Pauschbetrag}

Personen, denen laufende Hinterbliebenenbezüge (z. B. nach dem Bundesversorgungsgesetz oder aus der gesetzlichen Unfallversicherung) bewilligt worden sind, erhalten auf Antrag einen Pauschbetrag von 370 Euro (Jahresbetrag). Für die Übertragung des einem Kind zustehenden Pauschbetrags auf die Eltern oder Großeltern gelten die gleichen Grundsätze wie bei der Übertragung des Behinderten-Pauschbetrags $(\rightarrow$ Seite 21$)$.

Der Pauschbetrag wird auch gewährt, wenn das Recht auf die Bezüge ruht oder der Anspruch auf die Bezüge durch Zahlung eines Kapitals abgefunden worden ist.

\subsubsection{Weitere außergewöhnliche Belastungen}

Darüber hinaus kommen z. B. folgende Aufwendungen als außergewöhnliche Belastungen unter Berücksichtigung der zumutbaren Belastung $(\rightarrow$ Nr. 2.3) in Betracht:

- Bestattungskosten eines nahen Angehörigen, soweit sie nicht aus dem Nachlass bestritten werden können und nicht durch Ersatzleistungen gedeckt sind; nicht abziehbar sind Aufwendungen für die Bewirtung von Trauergästen und für Trauerkleidung

Aufwendungen zur Wiederbeschaffung oder Schadensbeseitigung für existentiell notwendige Gegenstände wie Wohnung, Hausrat und Kleidung, die durch ein unabwendbares Ereignis (Unwetter, Brand, Hochwasser) zerstört oder beschädigt wurden. Es müssen tatsächlich finanzielle Aufwendungen entstanden sein; ein bloßer Schadenseintritt reicht nicht aus. Außerdem werden die Aufwendungen nur insoweit berücksichtigt, als sie den Wert des Gegenstandes vor dem schädigenden Ereignis nicht übersteigen (verlorener Aufwand). Ein Abzug scheidet aus, sofern zumutbare Schutzmaßnahmen unterlassen oder eine allgemein zugängliche und übliche Versicherungsmöglichkeit nicht wahrgenommen wurden.

\subsection{Zumutbare Belastung}

Bei den außergewöhnlichen Belastungen allgemeiner Art werden die tatsächlichen Aufwendungen nur berücksichtigt, soweit sie eine Zumutbarkeitsgrenze, die „zumutbare Belastung" übersteigen. Diese ist nach dem Familienstand, der Kinderzahl und dem Gesamtbetrag der Einkünfte in drei Stufen gestaffelt. Nur der Teil des Gesamtbetrags der Einkünfte, der die jeweilige Betragsstufe übersteigt, wird mit dem jeweils höheren Prozentsatz belastet. 
bei einem Gesamtbetrag der Einkünfte

bis 15.340 Euro über 15.340 Euro bis 51.130 Euro
Personen, die einzeln zur Einkommensteuer veranlagt werden, ohne Kinder

Verheiratete und andere Personen mit Splitting-Verfahren, ohne Kinder

Steuerpflichtige mit 1 Kind oder 2 Kindern

Steuerpflichtige mit 3 oder mehr Kindern
5

4

2

1 über 51.130 Euro

6

7

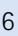

4 2
Beispiel:

Frau Meier, zwei Kinder, sind im Jahr 2018 zwangsläufige Krankheitskosten in Höhe von 2.000 Euro entstanden. Der Gesamtbetrag der Einkünfte beträgt 30.000 Euro.

Stufe 1: bis 15.340 Euro $\times 2 \%$ $=306$ Euro

Stufe 2: (15.341 Euro bis 30.000 Euro $=)$

14.660 Euro $\times 3 \%$ $=439$ Euro zumutbare Belastung (= Zumutbarkeit; 306 Euro + 439 Euro) 745 Euro abziehbar als außergewöhnliche Belastung (2.000 Euro - 745 Euro =)

1.255 Euro gleichgestellt sind Personen, denen zum Unterhalt bestimmte inländische öffentliche Leistungen (z. B. Arbeitslosengeld II) mit Rücksicht auf die Unterhaltsleistungen des Steuerpflichtigen ganz oder teilweise nicht gewährt werden (z. B. Partner einer eheähnlichen Lebensgemeinschaft). Bei Zahlungen an den geschiedenen oder dauernd getrennt lebenden Ehegatten kommt entweder ein Abzug als Sonderausgaben $(\rightarrow$ Nr. 1.4) oder als außergewöhnliche Belastung in Betracht.

Weiter müssen folgende Voraussetzungen vorliegen:

\subsection{Außergewöhnliche Belastungen in besonderen Fällen}

\subsubsection{Unterhaltsaufwendungen}

Aufwendungen für den Unterhalt und eine etwaige Berufsausbildung einer dem Steuerpflichtigen oder seinem Ehegatten oder Lebenspartner gegenüber gesetzlich unterhaltsberechtigten Person (z.B. Eltern, Großeltern, Kinder, Enkel, geschiedener Ehegatte, Mutter eines nichtehelichen Kindes gegenüber dem Kindesvater nach § 1615I BGB) können als außergewöhnliche Belastung geltend gemacht werden. Den gesetzlich Unterhaltsberechtigten
Es müssen Aufwendungen für den typischen Unterhalt (z. B. Unterkunft, Kleidung, Ernährung sowie Aufwendungen für die Berufsausbildung) geleistet werden.

- Für die unterstützte Person darf niemand Anspruch auf Kindergeld oder einen Freibetrag für Kinder haben $(\rightarrow$ Info-Broschüre "Kinder im Steuerrecht").

- Die unterstützte Person darf kein oder nur ein geringes eigenes Vermögen besitzen. Als geringfügig kann in der Regel ein Vermögen bis zu einem Verkehrswert von 15.500 Euro angesehen werden. Vermögensgegenstände des Unterhaltsempfängers, die zu seinem 
Hausrat gehören, und ein angemessenes eigengenutztes Hausgrundstück bleiben dabei außer Betracht.

Der Abzugsbetrag beträgt höchstens 9.000 Euro im Kalenderjahr 2018². Dieser Betrag erhöht sich um die Beiträge zu einer Basiskrankenversicherung oder gesetzlichen Pflegeversicherung $(\rightarrow$ Nr. 1.2.2) für die unterhaltsberechtigte Person, es sei denn, der Unterhaltsleistende kann diese Beiträge als Sonderausgaben abziehen. Der Höchstbetrag vermindert sich, soweit die eigenen Einkünfte und Bezüge der unterhaltenen Person den Betrag von jährlich 624 Euro übersteigen sowie um als Ausbildungshilfe bezogene Zuschüsse.

Die vorgenannten Beträge werden für jeden vollen Monat, in dem die Voraussetzungen nicht erfüllt sind, um je 1/12 gekürzt.

Unterhält der Steuerpflichtige mehrere Personen, die einen gemeinsamen Haushalt führen, so ist der Abzugsbetrag grundsätzlich für jede unterhaltene Person getrennt zu ermitteln. Der Gesamtbetrag der Unterhaltsleistungen ist nach Köpfen aufzuteilen und zwar auch, soweit unterhaltene Personen nicht unterhaltsberechtigt sind oder Kinder unterhalten wurden, für die Anspruch auf Kindergeld oder Freibeträge für Kinder besteht.

Tragen mehrere Steuerpflichtige die Aufwendungen für den Unterhalt bzw. die Berufsausbildung eines Unterhaltsempfängers, so wird der Abzugsbetrag diesen Steuerpflichtigen anteilig - entsprechend dem Anteil am insgesamt gezahlten Unterhalt - gewährt.

Lebt die unterstützte Person im Ausland, so können die Aufwendungen nur insoweit abgezogen werden, als sie nach den Verhältnissen des ausländischen Wohnsitzstaates notwendig und angemessen sind. Die o. g. Beträge werden daher ggf. nur zu 3/4, 1/2 oder $1 / 4$ angesetzt.

\subsubsection{Freibetrag zur Abgeltung des} Sonderbedarfs bei Berufsausbildung eines volljährigen Kindes

Der Freibetrag zur Abgeltung des Sonderbedarfs bei Berufsausbildung wird für auswärtig untergebrachte, volljährige Kinder in Berufsausbildung gewährt, für die der Steuerpflichtige Anspruch auf Kindergeld oder auf einen Freibetrag für Kinder hat. Ausführliche Informationen zur Höhe und Gewährung finden Sie in der $\rightarrow$ Info-Broschüre "Kinder im Steuerrecht".

2 Nach dem Entwurf des FamEntlastG sind folgende Änderungen 


\section{Berücksichtigung beim Lohnsteuerabzug}

Als Freibetrag können beim Lohnsteuerabzug alle unter Abschnitt 2 genannten außergewöhnlichen Belastungen und die unter $\rightarrow$ Nr. 1.4 bis 1.9 genannten Sonderausgaben, soweit sie den Sonderausgaben-Pauschbetrag von 36 Euro bzw. 72 Euro übersteigen, berücksichtigt werden.

Voraussetzung ist, dass die Sonderausgaben und außergewöhnlichen Belastungen ggf. zusammen mit Werbungskosten (über dem Arbeitnehmer-Pauschbetrag i. H. v. 1.000 Euro) die Antragsgrenze von 600 Euro überschreiten. Diese Antragsgrenze gilt nicht für die Eintragung der Pauschbeträge für behinderte Menschen und für Hinterbliebene.

Vorsorgeaufwendungen werden bei der Berechnung der Lohnsteuer durch eine Vorsorgepauschale $(\rightarrow$ Nr. 1.10) berücksichtigt. Deshalb ist insoweit kein Freibetrag möglich.

Wird ein Freibetrag eingetragen, besteht die Pflicht zur Abgabe einer Einkommensteuererklärung, wenn der im Kalenderjahr 2018 insgesamt erzielte Arbeitslohn die Grenze von 11.400 Euro bzw. bei Zusammenveranlagung 21.650 Euro $^{3}$ übersteigt.

Der Antrag auf Lohnsteuer-Ermäßigung ist beim zuständigen Finanzamt oder im Internet unter www.steuern.sachsen.de $\rightarrow$ Vordrucke $\rightarrow$ Bürger $\rightarrow$ Formular-Management-System der Bundesfinanzverwaltung - bzw. unter www.formulare-bfinv.de erhältlich.

Die Frist für eine Antragstellung beginnt am 1. Oktober des Vorjahres, für das der Freibetrag gelten soll. Damit sich der Freibetrag bereits beim Lohnsteuerabzug für Januar auswirken kann, muss der Antrag bis spätestens Ende Januar gestellt sein; der Freibetrag wird dann mit Wirkung ab 1. Januar als elektronisches Lohnsteuerabzugsmerkmal (ELStAM) berücksichtigt. Ein späterer Antrag hat zur Folge, dass das Finanzamt das Freibetragsvolumen auf die der Antragstellung folgenden Kalendermonate verteilen muss; der Freibetrag wird dann mit Wirkung vom Beginn des Monats berücksichtigt, der auf die Antragstellung folgt. Der Antrag auf Lohnsteuer-Ermäßigung muss spätestens bis zum 30. November für das laufende Kalenderjahr gestellt werden.

\footnotetext{
3 Nach dem Entwurf des FamEntlastG sind folgende Änderungen geplant:

in 2019: 11.600 Euro/22.050 Euro

ab 2020: 11.900 Euro/22.600 Euro
} 


\section{Hinweis auf weitere Broschüren}

Die Informationsbroschüren des Sächsischen Staatsministeriums der Finanzen "Steuertipps für Senioren", "Kinder im Steuerrecht" und "Rund um die Einkommensteuererklärung Was, Wie, Wo?" können Sie kostenlos beim Zentralen Broschürenversand der Sächsischen
Staatsregierung bestellen (Adresse: siehe Impressum).

Die Publikationen stehen auch im Internet unter www.publikationen.sachsen.de zum Download zur Verfügung. 


\section{Abkürzungsverzeichnis}

BAföG Bundesausbildungsförderungsgesetz

bzw. beziehungsweise

d.h. das heißt

ELStAM elektronisches Lohnsteuerabzugsmerkmal

EStG Einkommensteuergesetz

EU Europäische Union

ff. fortfolgende

GdB Grad der Behinderung

ggf. gegebenenfalls

i. H. v. in Höhe von

max. maximal

o. g. oben genannte

Pkw Personenkraftwagen

sog. sogenannte

SGB III Sozialgesetzbuch Drittes Buch

SGB IX Sozialgesetzbuch Neuntes Buch

SGB XI Sozialgesetzbuch Elftes Buch

SBG XII Sozialgesetzbuch Zwölftes Buch

u.a. unter anderem

z. B.

zum Beispiel

ZfA Zentrale Zulagenstelle für Altersvermögen

zzgl. zuzüglich 
